\title{
SuperPhy: predictive genomics for the bacterial pathogen Escherichia coli
}

\author{
Matthew D. Whiteside ${ }^{\dagger}$, Chad R. Laing ${ }^{*}{ }^{\dagger}$, Akiff Manji, Peter Kruczkiewicz, Eduardo N. Taboada \\ and Victor P. J. Gannon
}

\begin{abstract}
Background: Predictive genomics is the translation of raw genome sequence data into a phenotypic assessment of the organism. For bacterial pathogens, these phenotypes can range from environmental survivability, to the severity of human disease. Significant progress has been made in the development of generic tools for genomic analyses that are broadly applicable to all microorganisms; however, a fundamental missing component is the ability to analyze genomic data in the context of organism-specific phenotypic knowledge, which has been accumulated from decades of research and can provide a meaningful interpretation of genome sequence data.

Results: In this study, we present SuperPhy, an online predictive genomics platform (http://fz.corefacility.ca/ superphy/) for Escherichia coli. The platform integrates the analytical tools and genome sequence data for all publicly available E. coli genomes and facilitates the upload of new genome sequences from users under public or private settings. SuperPhy provides real-time analyses of thousands of genome sequences with results that are understandable and useful to a wide community, including those in the fields of clinical medicine, epidemiology, ecology, and evolution. SuperPhy includes identification of: 1) virulence and antimicrobial resistance determinants 2) statistical associations between genotypes, biomarkers, geospatial distribution, host, source, and phylogenetic clade; 3 ) the identification of biomarkers for groups of genomes on the based presence/absence of specific genomic regions and single-nucleotide polymorphisms and 4) in silico Shiga-toxin subtype.
\end{abstract}

Conclusions: SuperPhy is a predictive genomics platform that attempts to provide an essential link between the vast amounts of genome information currently being generated and phenotypic knowledge in an organismspecific context.

Keywords: Comparative genomics, Bioinformatics, Anti-microbial resistance, Virulence factors, Epidemiology, Population genomics, Software

\section{Background}

Whole-genome sequencing (WGS) of bacterial isolates generates the complete DNA sequence of each organism. WGS provides the greatest possible resolution of any typing method, the sequence is easily transferable, and its analyses can reveal important phenotypic insights such as the presence of virulence factors or antimicrobial resistance determinants. Current benchtop sequencers such as the Illumina MiSeq and the newly developed USB-sized Oxford Nanopore sequencer have made it possible for real-time WGS to be performed in

\footnotetext{
* Correspondence: chad.laing@canada.ca

${ }^{\dagger}$ Equal contributors

National Microbiology Laboratory @ Lethbridge, Public Health Agency of Canada, Lethbridge, AB T1J 3Z4, Canada
}

the laboratory as well as on the front-line, as was recently seen in the 2014 Ebola outbreak, and in managing a hospital outbreak of Salmonella [1-4].

WGS will likely replace current typing and sub-typing methods due to its low cost, high information content, portability, and speed of analyses. It is now being used in real-time for: the identification of the source of foodborne outbreaks [5], surveillance [6, 7], epidemiological investigations [7], industrial applications [8, 9], population studies [10,11], routine typing [12], regulation [13], providing point-of-care insight for clinicians [14, 15], informing veterinary practice [16], and helping inform public-health decisions [17].

WGS is now the de facto standard for bacterial strain analyses and the global community is coming together 
to help store and best utilize this rapid influx of information under the Global Microbial Identifier network (http://www.globalmicrobialidentifier.org/). This international effort currently involves 32 countries, many of which have their own national or regional programs to best utilize WGS data in public health, epidemiological and research contexts, such as the GenomeTrakR initiative of the Food and Drug Administration in the United States of America (http://www.fda.gov/Food/ FoodScienceResearch/WholeGenomeSequencingProgram WGS/), the Integrated Rapid Infectious Disease Analysis (IRIDA) platform in Canada (http://www.irida.ca/), and the Patho-NGen-Trace project within the European Union (http://patho-ngen-trace.eu/project/).

Recently, several platforms have emerged that attempt to provide additional context in addition to the raw WGS data. For instance PATRIC provides pre-computed analyses for public genomes, including annotation, protein families, antibiotic resistance identification and comparative pathway analysis [18]. MicroScope provides an expert-guided annotation pipeline, as well as comparative analyses based on shared gene content [19]. The Integrated Microbial Genomes (IMG) project is also a combined genome annotation and analysis platform, that additionally allows for genomic data submissions by the user [20]. BIGSdb allows local comparisons among genomes using a multi-locus sequence typing approach, and allows phenotypic data to be stored along with the genomic information [21]. The Harvest suite of tools allows for fast core-genome alignments and interactive visualizations for thousands of genomes [22]. Other platforms focus on a specific organism, such as Sybil, a platform for the comparative analyses of Streptococcus pneumoniae based on BLASTP searches [23].

The large initiatives that generate and collect the tensand hundreds-of thousands of genome sequences, and the platforms that host and analyze the public data provide an enormous benefit. Even though WGS and basic comparative analyses is commonplace, meaningful interpretation of the raw data in a phenotypic context, also known as predictive genomics, lags considerably behind [24]. Microbiologists often have organism-specific knowledge that can meaningfully inform the WGS data, but which is not incorporated into a generic analysis. The ability to interactively explore species-specific data that contains organism-specific knowledge from experts in the field is of tremendous value. A recent study on outbreak investigations using WGS also listed a main obstacle of routine adoption as 'a paucity of user-friendly and clinically focused bioinformatics platforms' [25]. While some components necessary for phenotypic prediction based on WGS data have been developed, there is currently no single integrated platform built to provide predictive genomic analyses for organism-specific end-users.
Here we present SuperPhy, a predictive genomics platform that brings organism-specific knowledge to comparative genomic analyses. SuperPhy incorporates knowledge from research on the pathogenesis and epidemiology of $E$. coli, as well as the tremendous amount of genotypic and phenotypic data that have previously been generated. This knowledge is used within SuperPhy to discover relationships among and about subgroups. It allows non-bioinformaticians to quickly analyze new data against the background of other sequenced $E$. coli, facilitating novel insights.

We have previously developed Panseq, software that performs comparative genomics in a pan-genome context, identifying differences in the accessory genome and single nucleotide variations within the core genome [26]. SuperPhy utilizes the pan-genomic output from Panseq to identify: 1) virulence and antimicrobial resistance determinants 2) epidemiological associations between specific genotypes, biomarkers, geospatial distribution, host, source, and other metadata in an interactive and explorable setting; 3 ) statistically significant clade-specific genome markers (presence/absence of specific genomic regions, and single-nucleotide polymorphisms) for bacterial populations; and 4) in silico Shiga-toxin subtyping for genomes that possess stx genes.

SuperPhy allows the submission of genomes in a private or public context and is continually updated with the influx of public E. coli data from GenBank, allowing researchers to quickly analyze and compare new genomes with other publicly available sequenced $E$. coli strains. Predictive genomics provides an essential link between the vast numbers of genomes currently being generated and organism-specific phenotypic knowledge.

\section{Platform features}

\section{Navigation and overview}

The layout of the SuperPhy website (https://lfz.corefacility.ca/superphy) provides universal and quick access to the major components of the platform: 'Group Analyses' provides an interactive environment for comparing groups of strains based on metadata types or usercreated strain-groupings, and determining statistically significant biomarkers for these groups (both the presence/absence of genomic regions and SNPs); 'VF and AMR' provides an ontology of both virulence genes and AMR determinants, and the ability to select groups of genomes and factors based on the provided ontologies. Output includes a summary of the presence/absence of selected VF and AMR factors among the strains of interest; 'Group Browse' provides an interface to examine groups of strains, and their distribution in both a geospatial and phylogenetic context simultaneously; 'My Data' provides an interface for uploading and modifying user-submitted genomes that are available only to the 
user; 'Home' provides a landing page and an overview of the features of the site. Additionally, an in-depth examination and report on an individual strain, including all known metadata, Shiga-toxin subtype (if applicable), phylogenetic and geospatial information, and a summary of virulence factor and anti-microbial resistance determinants can be accessed by selecting 'detailed information' from any genome in the platform.

\section{Strain selection}

SuperPhy provides three methods of selecting E. coli genomes for analyses that are consistent across the site: list-, tree-, and map-based selections. The platform is based heavily on metadata, and as such provides a unified metadata control panel that displays the metadata fields and their associated values for each genome across each of the three views. The metadata control panel also allows filtering and selecting genomes that match given metadata criteria.

1) List-based selection provides a table-based interface to the genomes and their metadata, with private and public genome sets afforded their own sections.

2) Tree-based selection provides an interactive phylogeny that can be manipulated to expand/ contract clades, and from which clade and individual genome selection can be made. Metadata is appended to each leaf node of the tree, and branches containing more than one genome have the metadata for the entire branch summarized as an interactive bar-chart that displays the frequency of values within selected metadata categories. This summary is an excellent way to visually discern clade differences, and allows an effective representation of thousands of genomes in tree form that would otherwise be intractable. An example of the phylogenetic tree with metadata clusters is shown in Fig. 1.

3) Map-based selection provides a Google Maps interface to geospatial genome selection, along with a table-view of the metadata for the genomes in the map. Just as in the list-based view, the displayed metadata fields for each genome can be changed, and used to filter the displayed genomes. As an example, we show the map when a user searches for 'United Kingdom' in Fig. 2.

\section{Website usage tutorials}

The main pages of the SuperPhy platform include a guided tutorial introduction using the IntroJS plugin (https://usablica.github.io/intro.js/). This tutorial provides a walk-through of all the major features and how to use them, and is activated by clicking the large red 'Introduction' button located on each page.

\section{Implementation}

\section{Webserver application and database}

Genome data and analyses are administered using a PostgreSQL 9.3 database with a schema adapted from the Generic Model Organism Database (GMOD) Chado schema [27]. The Chado relational database schema uses a flexible, ontology-centric approach to organizing biological entities, relationships, properties and analyses. Entries in generic tables are assigned types using a mutable, controlled vocabulary. By not defining entity types directly into the relational layer, the database can be highly adaptable and can grow to add new analyses or biological data.

The application layer for the SuperPhy website is build using the Model-View-Controller (MVC) Perl CGI::Application framework (http://www.cgi-app.org/). The phylogenetic tree display and interaction is built on top of the Data Driven Documents (D3) JavaScript library (http://d3js.org/). Geospatial views are built using the Google Maps JavaScript API v3 (https://developers.google. com/maps/documentation/javascript/). Group comparisons are processed and displayed using the RStudio Shiny web application framework for R [28].

The webserver application code base, database schema and public data are hosted on Github at https://github. com/superphy/version-1.

\section{Access to uploaded data}

Users can upload genomes and metadata and choose between three access levels to govern their use: 'public' information is available to all users; 'private' information is only available for the genome uploader and additional users they select; and 'private until a specified date' data is released to 'public' data after a specified date. Users may also designate other registered users for whom the data will be available. Private data is accessible only to designated users, but can be combined with public data for user-specific analyses. Users can create custom genome-groups that can be saved, and all results may be downloaded for offline analyses.

Uploaded data undergo a series of checks to ensure the quality of the data. Data are rejected if any of the following conditions are met: 1) Greater than 1000 contigs; 2) Genome size less than $3 \mathrm{Mbp}$ or greater than $7.5 \mathrm{Mbp}$; 3) Invalid nucleotide characters (all IUPAC characters are valid); 4) The MD5 checksum of the concatenated contigs already exists in the database; 5) The SNP string for the pan-genome alignment is identical to another strain in the database.

Uploaded genomes undergo two checks to ensure the data are of a minimum quality, and that the genomes being uploaded contain markers that were found to be present only in genomes of $E$. coli. We initially identified genomic regions present in at least $70 \%$ of the genomes, 


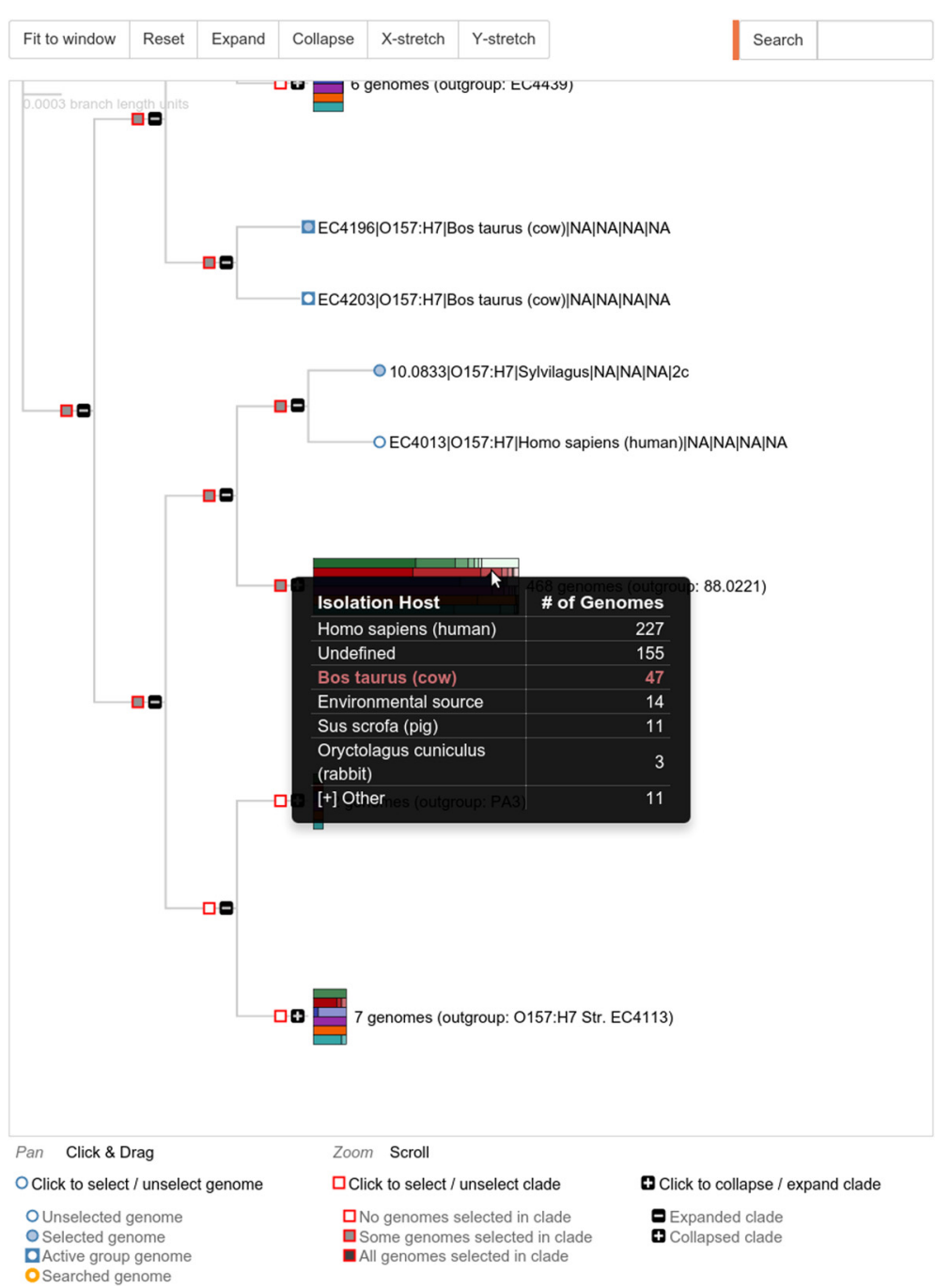

Fig. 1 Interactive phylogeny with metadata. A screen capture showing tree-based selection from an interactive phylogeny that can be manipulated to expand/contract clades, and from which clade and individual genome selections can be made. Metadata is shown appended to each leaf node of the tree, and branches containing more than one genome have the metadata for the entire branch summarized as an interactive bar-chart. Each colored bar represents a metadata category, which is summarized in table form when highlighted; here the red bar representing Isolation Host is shown with a frequency table of hosts. Metadata represented as bars are as follows: Green:Serotype, Red:Isolation Host, Blue:Isolation Source, Purple:Symptoms/Disease, Orange:Stx1-subtype, Teal:Stx2-subtype

referred to as the "conserved core". All genomes are considered to be E. coli if: 1) they contain at least 1500 conserved core regions, and 2) The presence of at least three E. coli species-specific regions. The derivation of these markers is presented in the 'Pan-genome' subsection of the 'Example analyses'.

\section{Acquisition of public Escherichia coli genomes}

SuperPhy is continually and automatically updated with closed and draft genomes of Escherichia coli from GenBank using the script https://github.com/superphy/version-1/ Sequences/ncbi_downloader.pl. All metadata present in the GenBank submissions are extracted automatically using the script https://github.com/superphy/version-1/ Sequences/genbank_to_genodo.pl. For the initial bulk upload, a second phase of manual curation was carried out to ensure all available metadata was included, even if it was stored in a non-standard way during the initial submission. The complete list of 1641 public E. coli genomes present in the SuperPhy database at the time of manuscript preparation, along with all extracted metadata is available at (https://github.com/superphy/version-1/ Data/metadata_table.csv). A summary of the metadata fields used in SuperPhy, as well as the percentage of the 


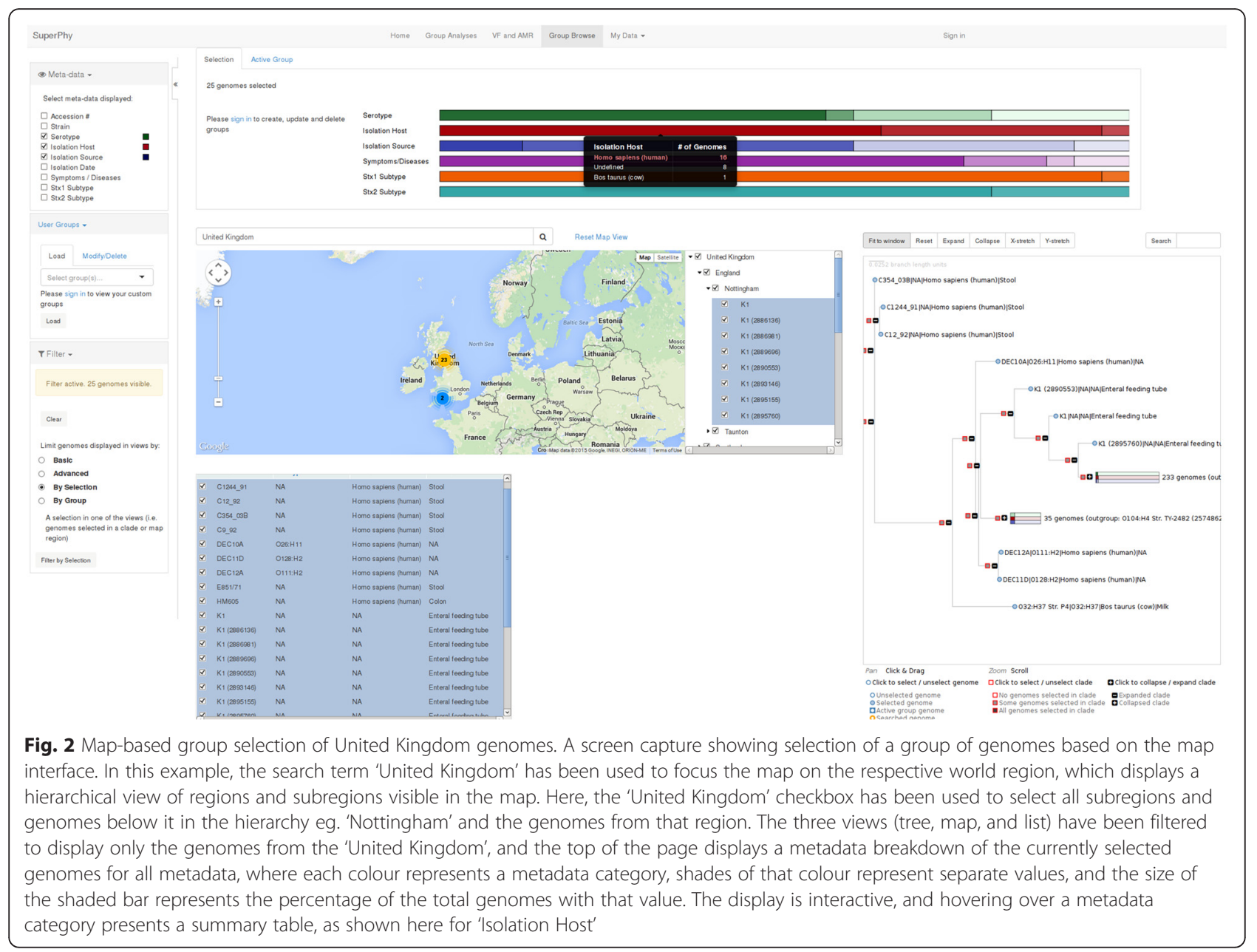

public genomes containing information for a particular metadata category is presented in Table 1.

\section{Comparative genomic analyses}

Our pan-genomic analyses tool, Panseq is used for the background comparative analyses [26]. It iteratively adds new genomic sequences, and compares them to those already stored in the platform. This computational approach allows a continuous influx of new sequence data without large time or memory requirements. In this way, the complete pan-genome of all sequences in the database is determined. Annotations for these regions are determined by querying the GenBank NR protein database via BLASTx.

Differences in the accessory genome and the single nucleotide variation in the core genome are obtained and used by SuperPhy in downstream applications including the construction of discriminatory and robust phylogenies, and in the pre-computed data for bio-marker identification among groups of genomes.

\section{Tree construction}

SuperPhy provides a global phylogenetic tree that is updated to include all $E$. coli genomes currently in the database. An initial phylogenetic tree for SuperPhy was constructed using conserved genomic regions from the

Table 1 The percentage of genomes that contain metadata for each of the metadata fields in the initial public data set of 1641 E. coli in the SuperPhy database

\begin{tabular}{ll}
\hline Metadata field & Percentage \\
\hline Location & 85 \\
Host & 79 \\
Date of Isolation & 63 \\
Source & 52 \\
Serotype & 44 \\
Stx2 subtype & 23 \\
Stx1 subtype & 18 \\
Disease syndrome & 6 \\
\hline
\end{tabular}


1641 E. coli genomes obtained from GenBank. The conserved regions were aligned using Muscle [29] and input into FastTreeMP to build a minimum-evolution tree [30]. To achieve sufficient resolution in branch lengths to disambiguate strains, the double-precision version of FastTree was used [30]. As new genomes are uploaded to SuperPhy, they are incorporated into the multiple sequence alignment and a new tree is rebuilt, which becomes the tree used for all analyses within the SuperPhy platform.

\section{Virulence and anti-microbial resistance markers}

The presence/absence of virulence and AMR genes are computed using Panseq. The non-redundant query set of AMR genes from the Comprehensive Antibiotic Resistance Database (CARD) [31] is used for in silico AMR determinant screening. All AMR genes are organized and stored in the database according to their CARDassigned Antibiotic Resistance Ontology annotation to aid in identifying the presence of different antimicrobial resistance mechanisms. The virulence gene database was constructed by obtaining all gene alleles of known virulence factors for $E$. coli from the Virulence Factor Database [32], supplemented with additional virulence factors from 'Escherichia coli: Pathotypes and Principles of Pathogenesis, 2nd Ed.' [33], and additional published literature, which effectively doubled the number of virulence factors in the database. To avoid duplication of factors, all AMR and virulence factor sequences were clustered based on similarity using BLASTclust with default settings; the longest allele was selected for each gene, except in cases where sequence similarity was less than $90 \%$, in which case multiple alleles were included [34].

In addition to providing the presence/absence of virulence and AMR factors, SuperPhy stores the sequence of the individual alleles for each genome, and constructs a phylogeny based on each single gene. This allows one to compare the relationships among genomes based on a single virulence or AMR attribute and to examine the sequence variation of the gene at the individual base level, as the multiple sequence alignment (MSA) can also be displayed, as shown in Fig. 3.

\section{Group comparisons}

The statistical identification of markers that differ between groups based on both single nucleotide polymorphisms and the presence/absence of genomic loci is implemented using a two stage approach: 1) To rapidly assess the thousands of possibilities, the 'approximate' vectorized Fisher's Exact Test (FET) from the $\mathrm{R}$ corpora package is calculated (http://cran.r-project.org/ web/packages/corpora/index.html), following which the exact FET value is computed for the 100 most-

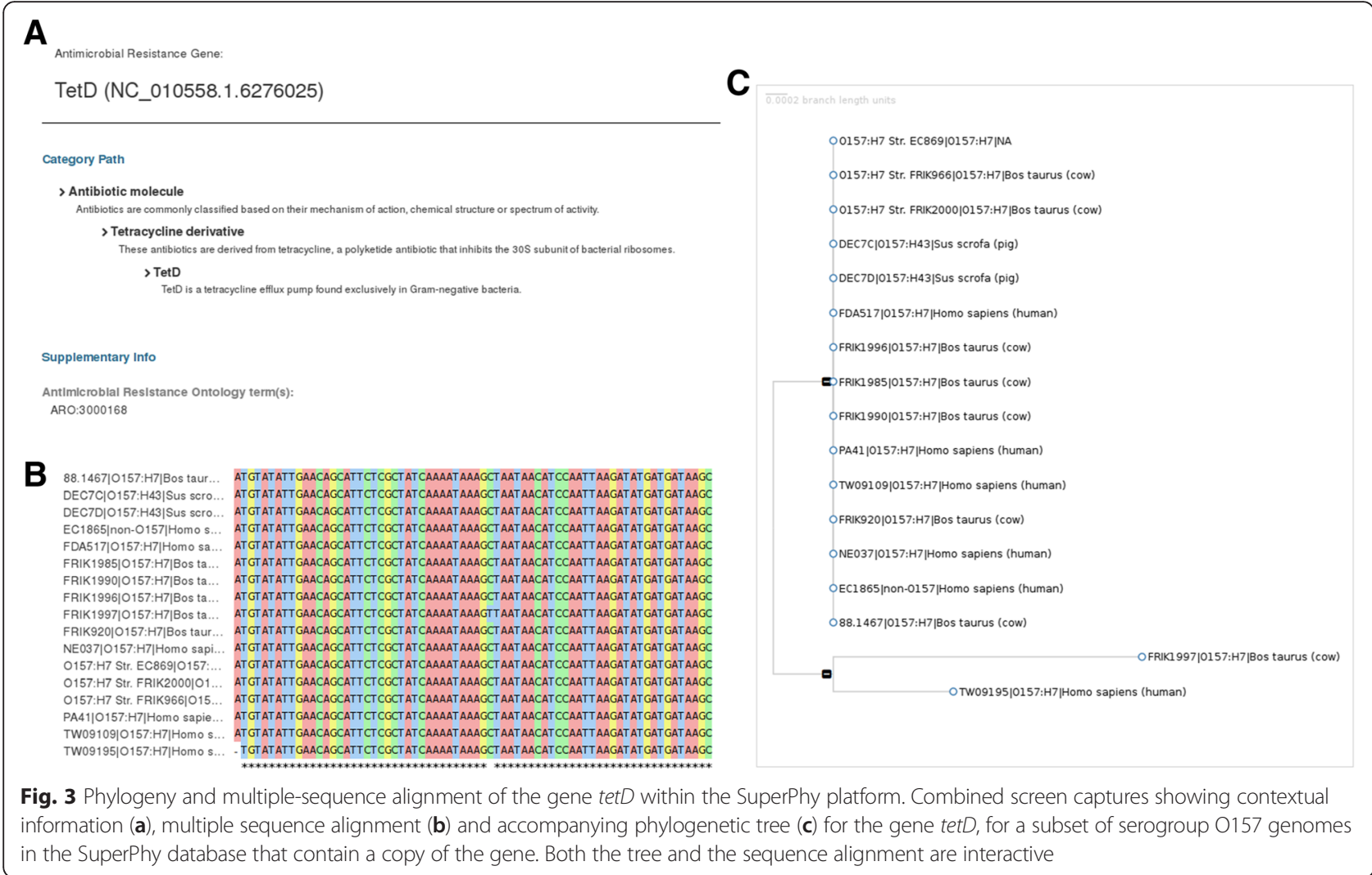




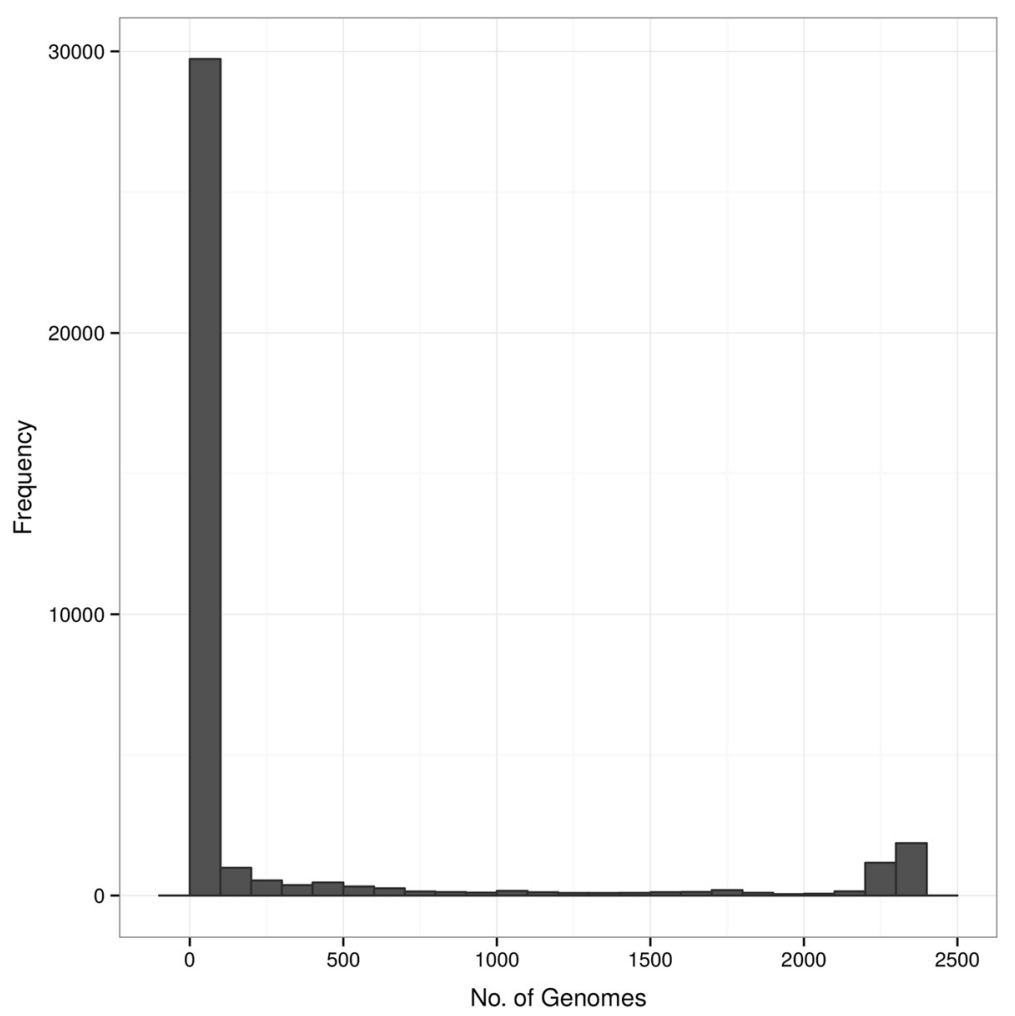

Fig. 4 The pan-genome distribution among 2324 E. coli genomes. The pan-genome distribution of 2324 E. coli genomes as 1000 bp genomic segments. The majority (29.7Mbp) of the $37.44 \mathrm{Mbp}$ pan-genome is present in fewer than 100 genomes, with the core genome size (present in at least 2300 genomes) observed to be $1.86 \mathrm{Mbp}$. Only $5.84 \mathrm{Mbp}$ of the pan-genome was found in greater than 100 genomes, but fewer than 2300 genomes. Of these 2324 genomes, only 1641 had metadata beyond the name of the strain

significant results using the FET function from the base $\mathrm{R}$ statistical package [35]. The comparisons are corrected for multiple-testing using the false-discovery rate method of Benjamini and Hochberg. All singlenucleotide polymorphisms and genomic presence/absence data reside in the database, and require only the retrieval and $P$-value computation for the strains of interest for the real time analysis of genome markers.

The R Shiny interface is used for group creation and all metadata fields are pre-populated for all strains in the database. This makes comparing, for example, all human and non-human strains of a given serotype as simple as selecting groups based on the serotype and host metadata fields, and clicking the compare button. Additionally, custom groups of any genomes can be created and saved to a user-profile so they become available whenever the user is logged in. These custom groups can include private genomes available only to the logged-in user, in addition to any public genomes.

\section{Stx typing}

Shiga-toxin (Stx) subtype assignment, when a strain possesses a copy of one or more of stx 1 or stx2, is calculated based on a phylogenetic tree generated from concatenated and aligned a and $\mathrm{b}$ subunits for each of Stx1 and Stx2. Clades specific to a Shiga-toxin subtype were identified based on the scheme presented by Scheutz et al. (2012) [36]. Membership in these predefined clades is used to identify the subtype of the toxin gene; those strains that fall outside of known subtype clades are marked as unknown. Multiple sequence alignments of the Stx genes are stored in the database for reference and comparison.

\section{Geospatial visualization}

The geospatial visualizations provide an interactive map interface for selecting and and searching genomes and groups of genomes. SuperPhy leverages Google Maps along with the companion Javascript library, Google Maps API (V3).

Genome location data is geocoded for latitude and longitude during the process of adding a new strain to the platform. To reduce the computational overhead in rendering thousands of genome map markers, the marker clustering algorithm MarkerClusterPlus for Google Maps V3 (http://google-maps-utility-library-v3.googlecode.com/ svn/trunk/markerclustererplus/docs/reference.html) was implemented. Locations within a distance of 60 pixels on 
the map are clustered into a single marker rendered at the geometric center of the cluster, and a count of the number of genomes is displayed.

All geospatial views are accompanied by a dynamic and sortable table of genome metadata that is by default sorted by country. Users also have the option of sorting by province, state and city. The table is dynamic and updates to display information for the genomes visible on the map. Locations for each E. coli strain can be downloaded for offline manipulation.

\section{Continuous integration}

The user community is able to provide feedback as the platform evolves in the form of feature requests and bug reports using the 'Issues' section at https://github.com/ superphy/version-1/issues. This will ensure the platform evolves in a way that is most beneficial to those who use it.

\section{Results and discussion Pan-genome}

At the time of writing, 2324 publicly available E. coli genomes from GenBank had been analyzed for incorporation into the SuperPhy platform [37]. E. coli is a ubiquitous, Gram-negative bacterial species found in the intestines of healthy mammals, with only a small subset causing disease in humans or animals [38]. The population structure of $E$. coli was initially described as being broadly distributed among four large and two smaller phylogenetic groups $[39,40]$. Previous studies have found that the species has an open pan-genome, meaning that the addition of new genomes is likely to add additional genes to the pool [41]. The pan-genome of $E$. coli is highly variable, with around $80 \%$ of an individual genome comprised of accessory genes and the remainder from the shared core genome [42]; a stable proportion of approximately 4000 genes are present in at least $50 \%$ of the genomes [43].

The pan-genome distribution of these $2324 E$. coli genomes as $1000 \mathrm{bp}$ genomic segments is presented in Fig. 4. As can be seen, the majority (29.7 Mbp) of the 37.44 Mbp pan-genome is present in fewer than 100 genomes, with the core genome size (present in at least 2300 genomes) observed to be $1.86 \mathrm{Mbp}$. Only 5.84 Mbp of the pan-genome was found in greater than 100 genomes, but fewer than 2300 genomes. Based on these results, we selected a 'conserved core' of 3598 genomic regions, defined as those present in at least $70 \%$ of the 2324 genomes. The conserved core is used within SuperPhy to identify SNPs that are used in phylogenetic tree building, as well as in the quality filtering of uploaded genomes.

Additionally, we endeavored to identify genomic regions that were specific to the species $E$. coli. To achieve this we screened the 'conserved core' against genomes from a subset of $E$. coli and other bacterial species, the results of which are presented in Table 2. The E. coli genomes contained more of the 'conserved core' regions than any of the other genomes examined, although genomes from Shigella spp. contained nearly as many, which is not surprising given that Shigella spp. has long been known to be very similar to $E$. coli [44]. Recent work using the analyses of whole genome sequence data of both Shigella spp. and E. coli showed Shigella spp. to form three separate monophyletic clades within the $E$. coli species [45], and that there was a mixing of traditional Shigella spp. within these clades. The analyses that we performed in the current study to find $E$. coli specific regions treated Shigella spp. as distinct from $E$. coli; had we considered them as sub-groups within $E$. coli, the number of species-specific markers would likely have increased.

The results shown in Table 2 were filtered based on the distribution among these 19 genomes to identify genomic regions present in only the E. coli genomes, resulting in 33 candidates; the raw data table is available at https:/github.com/superphy/version-1/Sequences/ genome_content_panseq/binary_table.txt. These 33 candidates were screened against the GenBank 'nr' and 'WGS'

Table 2 The number of conserved core genomic regions present in 19 selected bacterial genomes, from the total 3598 conserved core genomic regions found in at least $70 \%$ of the 2324 E. coli genomes examined

\begin{tabular}{ll}
\hline Genome & $\begin{array}{l}\text { No. 'conserved core' } \\
\text { genes }\end{array}$ \\
\hline E. coli O103:H2,12009 & 3563 \\
E. coli O157:H7, EDL933 & 3557 \\
E. coli K-12, MG1655 & 3550 \\
E. coli, UMN026 & 3483 \\
E. coli O7:K1, CE10 & 3448 \\
E. coli O83:H1, NRG 857C & 3289 \\
Shigella sonnei, 53G & 3259 \\
Shigella flexneri 2002017 & 3148 \\
Shigella boydii, CDC 3083-94 & 2965 \\
Shigella dysenteriae, 1617 & 2683 \\
Escherichia fergusonii ATCC 35469 & 1619 \\
Salmonella enterica subsp. Enterica serovar & 95 \\
Typhimurium str. 14028S & \\
Citrobacter rodentium, ICC168 & 77 \\
Klebsiella oxytoca, E718 & 50 \\
Klebsiella pneumoniae subsp. Pneumoniae, 1084 & 50 \\
Klebsiella variicola, At-22 & 46 \\
Escherichia blattae, DSM 4481 & 27 \\
Staphylococcus aureus, 04-02981 & 0 \\
Listeria monocytogenes, 07PF0776 & 0 \\
\hline
\end{tabular}


Table 3 The ten E. coli species-specific genomic regions identified in this study based on a total sequence identity of $90 \%$, their location in the K12 reference genome MG1655, the number out of 2324 E. coli genomes each region was found in, and their putative function based on the top scoring BLASTx hit

\begin{tabular}{|c|c|c|c|c|}
\hline Region ID & Start bp & End bp & No. genomes & Putative function \\
\hline 3160548 & 347258 & 346259 & 2238 & Propionate catabolism operon regulatory protein PrpR \\
\hline 3160296 & 537566 & 536567 & 2256 & 2-hydroxy-3-oxopropionate reductase \\
\hline 3160113 & 538566 & 537567 & 2248 & Allantoin permease \\
\hline 3159571 & 541565 & 540567 & 2275 & Purine permease ybby \\
\hline 3159389 & 542566 & 541567 & 2268 & Glycerate kinase \\
\hline 3158844 & 545665 & 544666 & 2261 & Allantoate amidohydrolase \\
\hline 3158667 & 546665 & 545666 & 2272 & Ureidoglycolate dehydrogenase \\
\hline 3159808 & 1588200 & 1587201 & 2171 & FimH protein \\
\hline 3160196 & 4411062 & 4410063 & 2261 & Hypothetical protein \\
\hline 3158082 & 4456632 & 4457631 & 2074 & Mur ligase family, glutamate ligase domain protein \\
\hline
\end{tabular}

databases using the 'bacteria' taxid to limit the search; the raw BLAST results are available at https:/github.com/ superphy/version-1/Sequences/genome_content_pan seq/UB0HWGTR015-Alignment.xml and https://github .com/superphy/version-1/Sequences/genome_content_ panseq/UD4GVA26015-Alignment.xml. Based on these queries using a $90 \%$ total sequence identity threshold, we removed all putative species-specific regions that were identified in genomes from bacteria other than $E$. coli, and were left with the ten species-specific regions presented in Table 3.

The correlation between the species-specific regions and the 'conserved core' regions among the $2324 \mathrm{E}$. coli genomes is presented in Fig. 5. As can be seen, not all species-specific markers were found in all strains; however, most E. coli genomes contained at least 8 of the

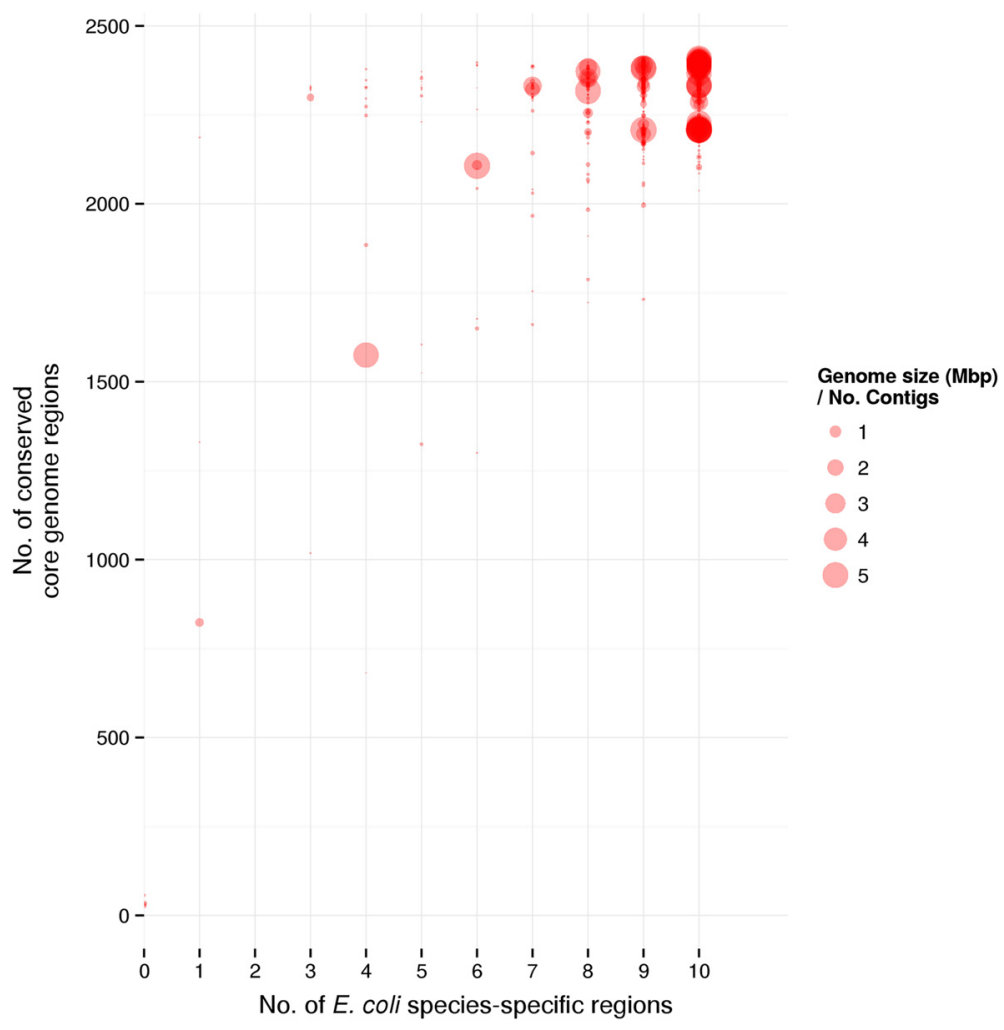

Fig. 5 Correlation between species-specific regions and genome quality. The correlation between the presence of the ten species-specific regions and the 3598 'conserved core' genomic regions identified in this study, among 2324 E. coli genomes. Genomes are plotted as dots where the size of the dot reflects genome quality, given by "genome size (Mbp)"/"No. contigs" 
markers and all contained at least 3 given the quality checks for assembled genomes previously described. A general trend was observed where genomes with higher ratios of 'Genome size'/'No. contigs' contained both more 'conserved core' regions and species-specific regions, indicating that the quality of genome assembly affects the number of genomic regions that can be identified at a given sequence identity threshold. Based on these results, any genome in the SuperPhy database is defined as E. coli if it possesses at least three of the species specific markers and at least 1500 of the conserved core genomic regions.

Of the 2324 genomes examined, only 1641 had metadata beyond the name of the strain. As such, the initial SuperPhy database contained only these 1641 genomes to facilitate a metadata driven approach to genomic analysis.

\section{Predictive markers for sub-groups}

A 'group' of bacteria can be defined in numerous ways, from spatially or temporally co-located strains, to those sharing biochemical utilization patterns, or those that occupy a clade of a phylogenetic tree. Regardless of how a group is defined, users are generally interested in defining characteristics that are predictive of the group, and can be used to discriminate its members from those of other related genomes. SuperPhy utilizes both the presence/absence of genomic regions, and SNPs within shared regions to define markers statistically predictive of a group. These identified biomarkers have potential downstream application in in silico diagnostics or simple wet-lab tests for the identified markers.

As an example, we utilized the 'Group Analyses' feature of SuperPhy to identify SNPs that were statistically predictive for $E$. coli of serotype O157:H7 with respect to those of all other E. coli. This is demonstrated in Fig. 6, where the SNPs are ranked from most- to leastsignificant. The marker ID for each SNP, the polymorphism being examined, the $p$-value, the false discovery rate adjusted $p$-value, and the presence/absence of each SNP for the two groups being examined are displayed. The marker ID provides a link to a 'SNP Information' page, which identifies the pan-genome region the SNP is found in, the allele frequency of SNPs for all genomes in the database, the putative function of the region given by the top BLAST hit, and an option to download detailed SNP information for each genome. The download includes the genomic location, allele, and upstream/ downstream sequences for all genomes in the database.

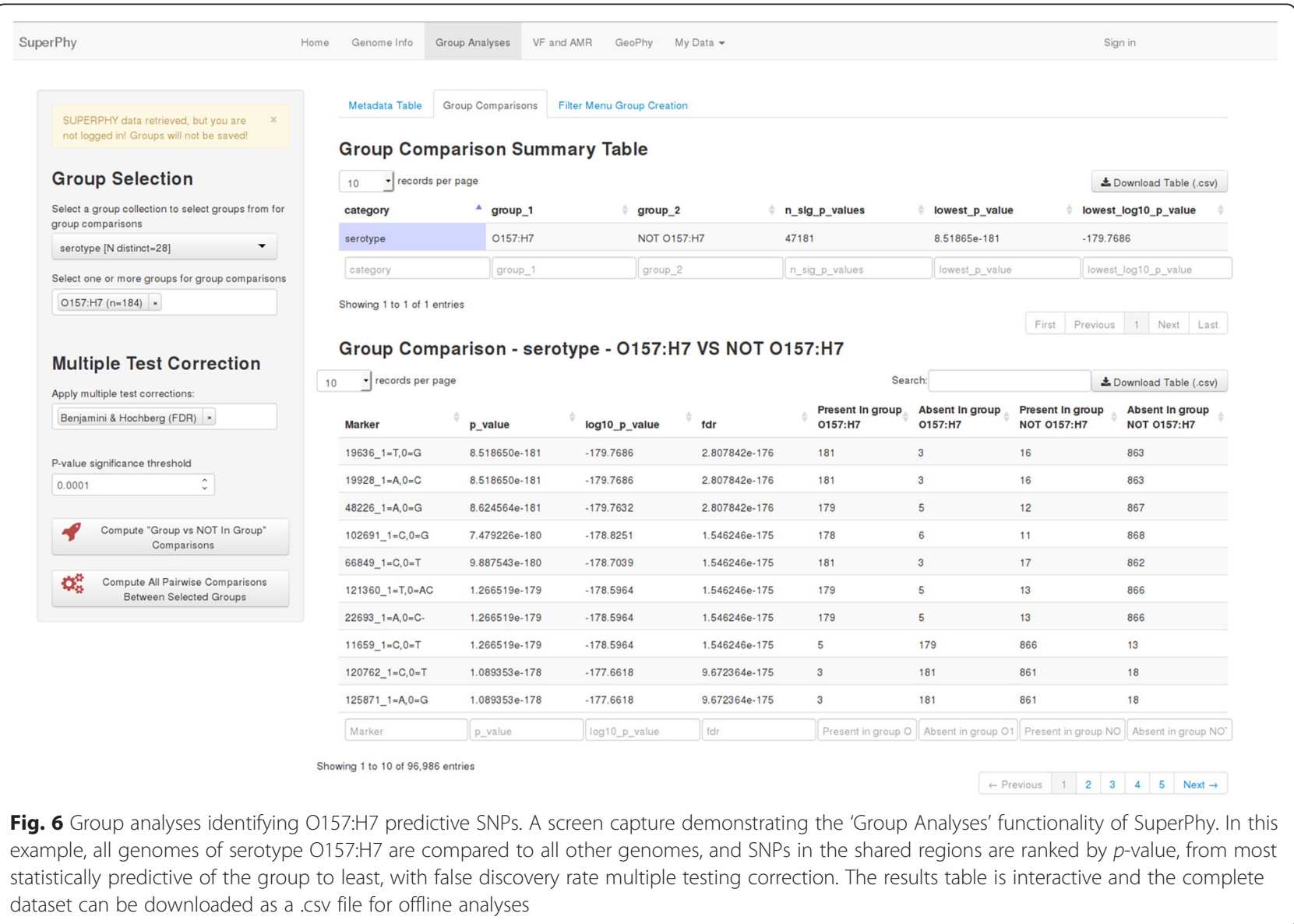



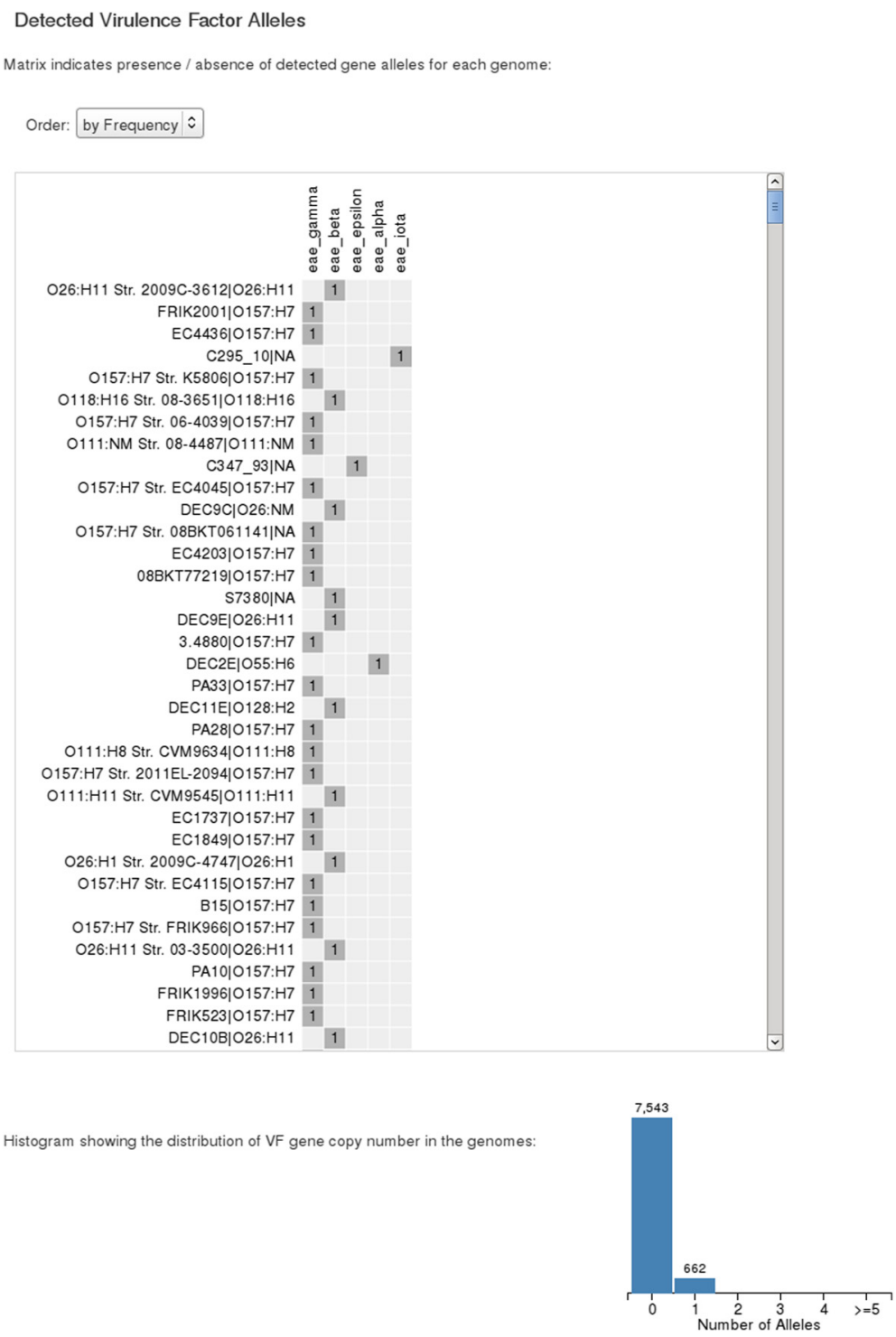

Fig. 7 Virulence factor analyses in SuperPhy. A screen capture showing the matrix representation of all genomes that contain an allele of the eae gene. The matrix contains more data than can be displayed in a single image, but it is interactive and scrollable, allowing the full matrix to be explored by the user. The metadata category 'Serotype' has been activated and can be seen as appended to the strain name in the row names of the matrix. The numbers within the matrix indicate the copy number of an allele within a genome, and empty boxes indicate the absence of an allele. The histogram displays the copy number of all genes searched for; in this case, the number of eae alleles

In addition to providing groups based on metadata categories such as serotype, and providing group vs. non-group comparisons, SuperPhy allows multi-way group vs. group comparisons. For example, if 'isolation host' is selected, then the categories 'Bos taurus (cow)', 'Homo sapiens (human)', and 'Environmental source' are used to generate comparisons between all combinations of the categories. This facilitates more rapid identification of group and sub-group predictive markers for the genomes being examined.

\section{Distribution of the eae gene}

Within the species $E$. coli, there are a subset of strains that attach to human intestinal epithelial cells via an attaching and effacing mechanism, the requisite apparatus for which is encoded in a genomic island known as the locus of enterocyte effacement (LEE) [46]. As an example of the 'VF and AMR' functionality within SuperPhy, we identified the distribution of the LEE gene eae among the 1641 public genomes in the SuperPhy database. All virulence factors are stored using controlled 
ontologies, which facilitate easy addition and retrieval of related data. The ontological category 'LEE-encoded TTSS effector' provided the eae alleles, and they were selected, along with all 1641 public genomes. The results are presented in an interactive matrix of gene presence/ absence, as well as allele copy number (Fig. 7). Within the 1641 genomes examined, 662 possessed any of the 11 known variants of the eae gene at a sequence identity cutoff of $90 \%$. Additionally, SuperPhy provides a table of the results for download, where subsequent offline manipulation is possible.

\section{Analyses of geographical and phylogenetic clusters}

The 'Group Browse' section of SuperPhy provides a means for selecting, filtering and exploring groups of genomes utilizing the three modes of genome selection, namely the tree, map and list views. These allows users to view geographical clusters in terms of their corresponding position in a phylogenetic tree. For example, using the map view, and the hierarchical listing of locations, all genomes with the isolation location of Santa Clara, California, United States were selected and their corresponding positions on the phylogenetic tree automatically highlighted, as shown in Fig. 8 . Here it is evident that although all six genomes were isolated from Santa Clara, California on the same day, the genomes do not form their own cluster on the phylogenetic tree. On the tree, all nodes that contain a selected genome are shown as blue-filled squares, while those that do not are white-filled squares. Similarly, all selected genomes appear on the tree as blue-filled circles, and those not selected as white-filled circles. The six selected genomes from Santa Clara are widely distributed throughout the tree (at this zoom level, they are not all visible). Genomes CS02 and CS06 are both visible, on separate branches of the tree, indicating they are less related to each other, and the other four genomes from Santa Clara, than several other $E$. coli genomes with which they group more closely.

This ability to quickly examine geographical strain clusters in a phylogenetic context would prove extremely useful in determining if a group of genomes from the same time and place originated from a single bacterial clone, as in an outbreak situation or in the routine surveillance of a location such as a food-processing plant, to determine whether bacterial isolates were that of a persistent strain.

Conversely, within SuperPhy one can also select a phylogenetic clade and have the geographical locations of all strains shown. The ability to break apart a cluster of strains that are related at the genome level into geographical and metadata categories has use in source tracking of strains, and in determining the geographical dissemination of bacterial clones over time. As an example, genomes from the serotype O104:H4 outbreak that occurred in Germany in 2011 were chosen. This

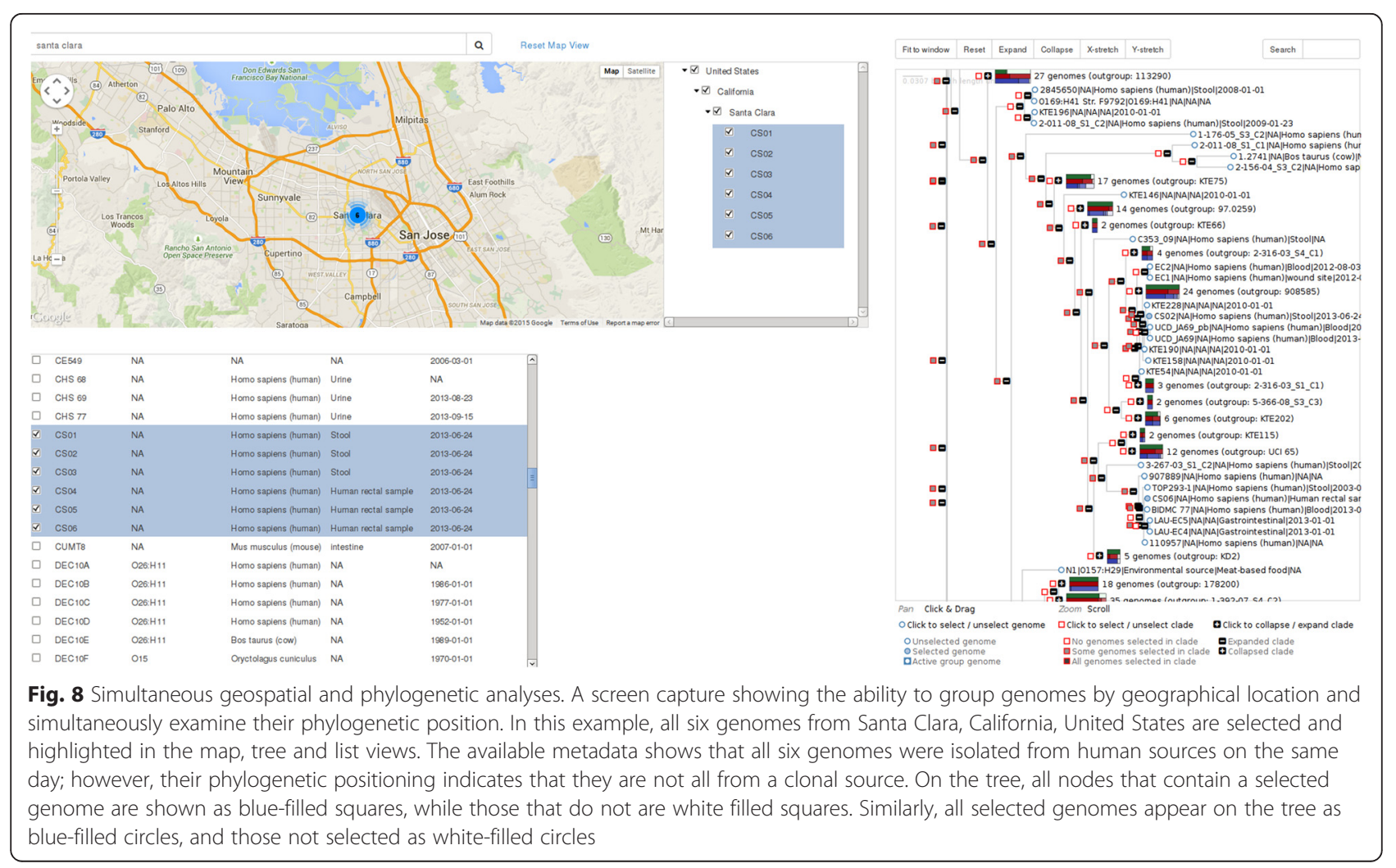


outbreak was the first caused by strains of O104:H4 that were found to have acquired the stx 2 gene through lateral gene transfer, which is thought to have been the contributing factor that led to the high rates of acute illness in healthy adults observed throughout the outbreak [47]. As can be seen in Fig. 9, the O104:H4 strains containing the stx 2 gene are nearly identical on the phylogenetic tree; however, the source of isolation of these bacteria, visible on the map, shows the dissemination of the bacterial clone from the German epicenter to countries such as Denmark, the United Kingdom, Canada, and the United states, which were determined to be travel-acquired infections.

\section{Conclusions}

Predictive genomics and platforms that easily facilitate it are poised to become the translation layer between the vast amounts of sequence data and biological knowledge in a specific domain that is needed to test hypotheses. SuperPhy allows users to make some of these genotype/ phenotype correlations, and platforms like it will become increasingly important in transforming raw genome data into useful knowledge.

Current work involves the addition of previously published in silico serotyping schemes to SuperPhy, and the expansion of the platform to include the bacterial pathogens Salmonella enterica and Campylobacter jejuni. Lastly, a representational state transfer (REST) application programming interface (API) is being designed to allow programmatic interaction with the SuperPhy platform, which will help ensure that SuperPhy does not become a data silo but can instead contribute to a dynamic and growing web of biological knowledge.

\section{Availability and requirements}

Project name: SuperPhy

Project home page: https://lfz.corefacility.ca/superphy Operating system(s): Platform independent (modern web-browser; the most recent Firefox or Chrome for best experience)

Programming languages: Perl, Coffeescript/Javascript, $\mathrm{R}$ License: Apache2

\section{Availability of supporting data}

The project is entirely open source under the Apache 2 license (https://www.apache.org/licenses/LICENSE-2.0). All code and any additional files referenced in the manuscript are available at the GitHub repository https://github.com/superphy/version-1.

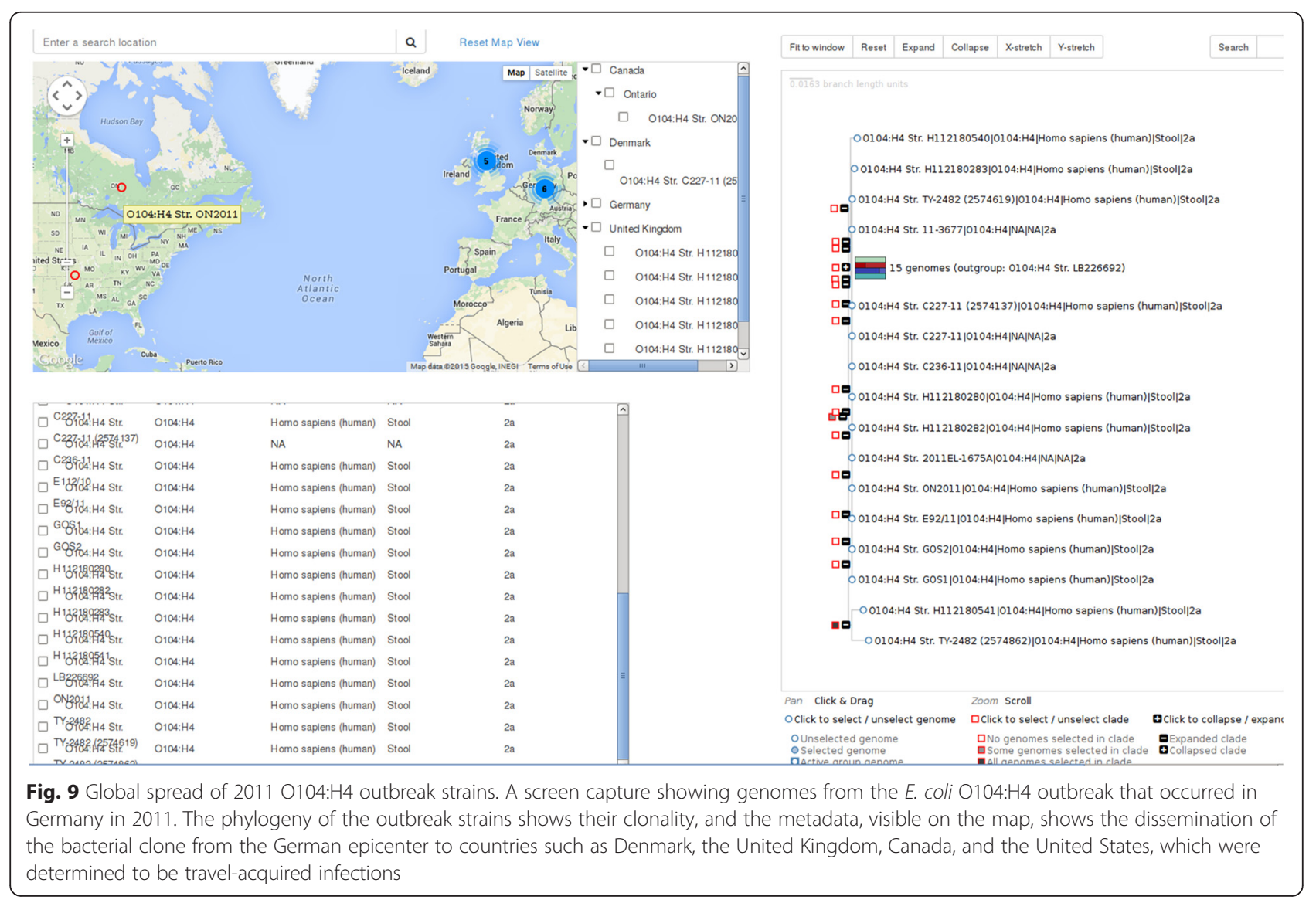




\section{Abbreviations}

AMR: anti-microbial resistance; CARD: comprehensive antibiotic resistance database; DNA: deoxyribonucleic acid; GMOD: generic model organism database; SNP: single-nucleotide polymorphism; Stx: Shiga-toxin; WGS: whole-genome sequencing.

\section{Competing interests}

The authors declare that they have no competing interests.

\section{Authors' contributions}

Designed the project: VPJG, CRL, MDW. Coded the platform: MDW, AM, CRL, PK. Wrote the manuscript: CRL, MDW, AM, VPJG. Contributed ideas; read, edited, and approved the manuscript: MDW, CRL, AM, PK, ENT, VPJG.

\section{Acknowledgements}

Thanks to Nicolas Tremblay for excellent metadata mining, Jason Masih for excellent front-end work, and Omar Zabaneh, Peter Shen, Michael Benediktson, and Waqar Gill for contributing to early versions of this project. This work is funded in part by the Public Health Agency of Canada and a grant from the Genomics Research and Development Initiative.

\section{Received: 29 September 2015 Accepted: 29 March 2016 Published online: 12 April 2016}

\section{References}

1. Jones B. Technology: nanopore sequencing for clinical diagnostics. Nat Rev Genet. 2015;16:68.

2. Gilchrist CA, Turner SD, Riley MF, Petri WA, Hewlett EL. Whole-genome sequencing in outbreak analysis. Clin Microbiol Rev. 2015;28:541-63.

3. Quick J, Loman NJ, Duraffour S, Simpson JT, Severi E, Cowley L, et al. Realtime, portable genome sequencing for Ebola surveillance. Nature. 2016 Feb 11;530(7589):228.

4. Quick J, Ashton P, Calus S, Chatt C, Gossain S, Hawker J, Nair S, Neal K, Nye K, Peters T, Pinna ED, Robinson E, Struthers K, Webber M, Catto A, Dallman TJ, Hawkey P, Loman NJ. Rapid draft sequencing and real-time nanopore sequencing in a hospital outbreak of Salmonella. Genome Biol. 2015;16:114.

5. Graham RMA, Doyle CJ, Jennison AV. Real-time investigation of a legionella pneumophila outbreak using whole genome sequencing. Epidemiol Infect. 2014;142:2347-51.

6. Zankari E, Hasman H, Kaas RS, Seyfarth AM, Agersø Y, Lund O, Larsen MV, Aarestrup FM. Genotyping using whole-genome sequencing is a realistic alternative to surveillance based on phenotypic antimicrobial susceptibility testing. J Antimicrob Chemother. 2013;68:771-7.

7. Cody AJ, McCarthy ND, Jansen van Rensburg M, Isinkaye T, Bentley SD, Parkhill J, et al. Real-time genomic epidemiological evaluation of human Campylobacter isolates by use of whole-genome multilocus sequence typing. J Clin Microbiol. 2013 Aug;51(8):2526-34.

8. Andreevskaya M, Johansson P, Laine P, Smolander O-P, Sonck M, Rahkila R, Jääskeläinen E, Paulin L, Auvinen P, Björkroth J. Genome sequence and transcriptome analysis of meat-spoilage-associated lactic acid bacterium lactococcus piscium MKFS47. Appl Environ Microbiol. 2015;81:3800-11.

9. Mazzaglia A, Studholme DJ, Taratufolo MC, Cai R, Almeida NF, Goodman T, Guttman DS, Vinatzer BA, Balestra GM. Pseudomonas syringae pv. actinidiae (PSA) isolates from recent bacterial canker of kiwifruit outbreaks belong to the same genetic lineage. PLoS ONE. 2012;7:e36518.

10. Nasser W, Beres SB, Olsen RJ, Dean MA, Rice KA, Long SW, Kristinsson KG, Gottfredsson M, Vuopio J, Raisanen K, Caugant DA, Steinbakk M, Low DE, McGeer A, Darenberg J, Henriques-Normark B, Beneden CAV, Hoffmann S, Musser JM. Evolutionary pathway to increased virulence and epidemic group A streptococcus disease derived from 3,615 genome sequences. Proc Natl Acad Sci. 2014;111:E1768-76.

11. Kopac S, Wang Z, Wiedenbeck J, Sherry J, Wu M, Cohan FM. Genomic heterogeneity and ecological speciation within one subspecies of Bacillus subtilis. Appl Environ Microbiol. 2014 Aug;80(16):4842-53.

12. Zhang $S$, Yin $Y$, Jones MB, Zhang Z, Kaiser BLD, Dinsmore BA, Fitzgerald C, Fields PI, Deng X. Salmonella serotype determination utilizing highthroughput genome sequencing data. J Clin Microbiol. 2015;53:1685-92.

13. Halachev MR, Chan JZ, Constantinidou Cl, Cumley N, Bradley C, Smith-Banks M, Oppenheim B, Pallen MJ. Genomic epidemiology of a protracted hospital outbreak caused by multidrug-resistant acinetobacter baumannii in Birmingham, England. Genome Med. 2014;6:70.
14. Grad YH, Lipsitch M. Epidemiologic data and pathogen genome sequences: a powerful synergy for public health. Genome Biol. 2014;15:538.

15. Jr WMD, Westblade LF, Ford B. Next-generation and whole-genome sequencing in the diagnostic clinical microbiology laboratory. Eur J Clin Microbiol Infect Dis. 2012;31:1719-26.

16. Biek R, O'Hare A, Wright D, Mallon T, McCormick C, Orton RJ, McDowell S, Trewby H, Skuce RA, Kao RR. Whole genome sequencing reveals local transmission patterns of mycobacterium bovis in sympatric cattle and badger populations. PLoS Pathog. 2012;8, e1003008.

17. Lemke AA, Harris-Wai JN. Stakeholder engagement in policy development: challenges and opportunities for human genomics. Genet Med. 2015 Dec; 17(12):949-57.

18. Wattam AR, Abraham D, Dalay O, Disz TL, Driscoll T, Gabbard JL, Gillespie JJ, Gough R, Hix D, Kenyon R, Machi D, Mao C, Nordberg EK, Olson R, Overbeek R, Pusch GD, Shukla M, Schulman J, Stevens RL, Sullivan DE, Vonstein V, Warren A, Will R, Wilson MJC, Yoo HS, Zhang C, Zhang Y, Sobral BW. PATRIC, the bacterial bioinformatics database and analysis resource. Nucleic Acids Res. 2013;42:D581-91.

19. Vallenet D, Belda E, Calteau A, Cruveiller S, Engelen S, Lajus A, Le Fevre F, Longin C, Mornico D, Roche D, Rouy Z, Salvignol G, Scarpelli C, Thil Smith AA, Weiman M, Medigue C. MicroScope-an integrated microbial resource for the curation and comparative analysis of genomic and metabolic data. Nucleic Acids Res. 2012:41:D636-47.

20. Markowitz VM, Chen I-MA, Palaniappan K, Chu K, Szeto E, Pillay M, Ratner A, Huang J, Woyke T, Huntemann M, Anderson I, Billis K, Varghese N, Mavromatis K, Pati A, Ivanova NN, Kyrpides NC. IMG 4 version of the integrated microbial genomes comparative analysis system. Nucleic Acids Res. 2013:42:D560-7.

21. Jolley KA, Maiden MC. BIGSdb: scalable analysis of bacterial genome variation at the population level. BMC Bioinformatics. 2010;11:595.

22. Treangen TJ, Ondov BD, Koren S, Phillippy AM. The Harvest suite for rapid core-genome alignment and visualization of thousands of intraspecific microbial genomes. Genome Biol. 2014;15(11):524

23. Riley DR, Angiuoli SV, Crabtree J, Hotopp JCD, Tettelin H. Using Sybil for interactive comparative genomics of microbes on the web. Bioinformatics. 2012;28:160-6.

24. Fricke WF, Rasko DA. Bacterial genome sequencing in the clinic: bioinformatic challenges and solutions. Nat Rev Genet. 2014;15:49-55.

25. Sherry NL, Porter JL, Seemann T, Watkins A, Stinear TP, Howden BP. Outbreak investigation using high-throughput genome sequencing within a diagnostic microbiology laboratory. J Clin Microbiol. 2013;51:1396-401.

26. Laing C, Buchanan C, Taboada EN, Zhang Y, Kropinski A, Villegas A, Thomas JE, Gannon VPJ. Pan-genome sequence analysis using panseq: an online tool for the rapid analysis of core and accessory genomic regions. BMC Bioinformatics. 2010;11:461

27. Mungall CJ, Emmert DB. A Chado case study: an ontology-based modular schema for representing genome-associated biological information. Bioinformatics. 2007:23:i337-46.

28. Racine JS. RStudio: a platform-independent IDE for $r$ and sweave. J Appl Econ. 2012;27:167-72.

29. Edgar RC. MUSCLE: multiple sequence alignment with high accuracy and high throughput. Nucleic Acids Res. 2004;32:1792-7.

30. Price MN, Dehal PS, Arkin AP. FastTree 2 - approximately maximumlikelihood trees for large alignments. PLoS ONE. 2010;5, e9490.

31. McArthur AG, Waglechner N, Nizam F, Yan A, Azad MA, Baylay AJ, Bhullar K, Canova MJ, Pascale GD, Ejim L, Kalan L, King AM, Koteva K, Morar M, Mulvey MR, O'Brien JS, Pawlowski AC, Piddock LJV, Spanogiannopoulos P, Sutherland AD, Tang I, Taylor PL, Thaker M, Wang W, Yan M, Yu T, Wright $\mathrm{GD}$. The comprehensive antibiotic resistance database. Antimicrob Agents Chemother. 2013;57:3348-57.

32. Chen L, Xiong Z, Sun L, Yang J, Jin Q. VFDB 2012 update: toward the genetic diversity and molecular evolution of bacterial virulence factors. Nucleic Acids Res. 2011:40:D641-5.

33. Donnenberg M, editor. Escherichia Coli: Pathotypes and Principles of Pathogenesis. 2nd edition. Amsterdam: Academic Press; 2013.

34. Altschul SF, Madden TL, Schäffer AA, Zhang J, Zhang Z, Miller W, Lipman DJ. Gapped BLAST and PSI-BLAST: a new generation of protein database search programs. Nucleic Acids Res. 1997;25:3389-402.

35. Statistical Computing RF. R: A language and environment for statistical computing. Vienna: R Development Core Team; 2005.

36. Scheutz F, Teel LD, Beutin L, Piérard D, Buvens G, Karch H, Mellmann A Caprioli A, Tozzoli R, Morabito S, Strockbine NA, Melton-Celsa AR, Sanchez 
M, Persson S, O'Brien AD. Multicenter evaluation of a sequence-based protocol for subtyping Shiga toxins and standardizing Stx nomenclature. Clin Microbiol. 2012;50:2951-63.

37. Benson DA, Cavanaugh M, Clark K, Karsch-Mizrachi I, Lipman DJ, Ostell J, et al. GenBank. Nucleic Acids Res. 2013 Jan;41(Database issue):D36-42.

38. Tenaillon O, Skurnik D, Picard B, Denamur E. The population genetics of commensal escherichia coli. Nat Rev Microbiol. 2010;8:207-17.

39. Selander RK, Caugant DA, Ochman H, Musser JM, Gilmour MN, Whittam TS. Methods of multilocus enzyme electrophoresis for bacterial population genetics and systematics. Appl Environ Microbiol. 1986;51:873-84.

40. Goullet P, Picard B. Comparative electrophoretic polymorphism of esterases and other enzymes in escherichia coli. J Gen Microbiol. 1989;135:135-43.

41. Medini D, Donati C, Tettelin H, Masignani V, Rappuoli R. The microbial pangenome. Curr Opin Genet Dev. 2005;15:589-94.

42. Lukjancenko O, Wassenaar TM, Ussery DW. Comparison of 61 sequenced escherichia coli genomes. Microb Ecol. 2010;60:708-20.

43. Gordienko EN, Kazanov MD, Gelfand MS. Evolution of pan-genomes of escherichia coli, shigella spp. and salmonella enterica. J Bacteriol. 2013;195: 2786-92.

44. Pupo GM, Lan R, Reeves PR. Multiple independent origins of Shigella clones of Escherichia coli and convergent evolution of many of their characteristics. Proc Natl Acad Sci U S A. 2000:97:10567-72.

45. Sahl JW, Morris CR, Emberger J, Fraser CM, Ochieng JB, Juma J, Fields B, Breiman RF, Gilmour M, Nataro JP, Rasko DA. Defining the phylogenomics of Shigella species: a pathway to diagnostics. J Clin Microbiol. 2015;53:951-60.

46. Croxen MA, Law RJ, Scholz R, Keeney KM, Wlodarska M, Finlay BB. Recent advances in understanding enteric pathogenic escherichia coli. Clin Microbiol Rev. 2013;26:822-80.

47. Mellmann A, Harmsen D, Cummings CA, Zentz EB, Leopold SR, Rico A, Prior K, Szczepanowski R, Ji Y, Zhang W, McLaughlin SF, Henkhaus JK, Leopold B, Bielaszewska M, Prager R, Brzoska PM, Moore RL, Guenther S, Rothberg JM, Karch $\mathrm{H}$. Prospective genomic characterization of the German enterohemorrhagic Escherichia coli 0104:H4 outbreak by rapid next generation sequencing technology. PLoS ONE. 2011;6, e22751.

\section{Submit your next manuscript to BioMed Central and we will help you at every step:}

- We accept pre-submission inquiries

- Our selector tool helps you to find the most relevant journal

- We provide round the clock customer support

- Convenient online submission

- Thorough peer review

- Inclusion in PubMed and all major indexing services

- Maximum visibility for your research

Submit your manuscript at www.biomedcentral.com/submit 